\title{
Novel Antennae for Luminescent Lanthanide Cations Emitting in the Visible and in the Near-Infrared: From Small Molecules to Polymetallic Lanthanide Containing Nanocrystals
}

\author{
Stéphane Petoud*
}

\begin{abstract}
Lanthanide cations have advantageous luminescence properties in the visible and in the near-infrared, such as high resistance to photobleaching which is a desirable property allowing long exposure of lanthanide compounds to excitation light without loss of luminescence intensity, and sharp emission bands whose wavelengths are not affected by experimental conditions, enabling efficient spectral discrimination from background fluorescence. Lanthanide cations also have long luminescence lifetimes that allow for temporal discrimination between sample and background. Due to these unique properties, lanthanide compounds could fulfill the requirements of luminescent reporters and sensors to be used in a broad range of applications, including biological analysis and biologic imaging. Free luminescent lanthanide cations have very limited absorption since $f-f$ transitions are forbidden. To overcome this limitation and to take advantage of their luminescence properties, lanthanide cations need to be located at close proximity to a sensitizer in order to generate an 'antenna' effect. In this review, we present and discuss different strategies and chemical systems that have been created and studied in the Petoud group in the Department of Chemistry of the University of Pittsburgh in order to develop novel antennae for lanthanide cations emitting in the visible and in the near-infrared. Two main avenues have been tested: a) The formation of luminescent monometallic lanthanide complexes using sensitizers based on derivatives of i) tropolonate, ii) azulene, iii) pyridine and iv) fluorene oligomers. b) The synthesis of systems where the number of lanthanide cations and the absorbance are maximized in order to enhance the number of photons per unit of volume in order to promote detection sensitivity. Different chemical approaches have been used to explore this strategy: i) polymetallic dendrimer complexes, ii) lanthanide containing semi-conductor CdSe nanocrystals, iii) tropolonate coated $\mathrm{Ln}^{3+}$-Doped $\mathrm{NaYF}_{4}$ nanocrystals and iv) metal-organic frameworks for polymetallic lanthanide complexes.
\end{abstract}

Keywords: Antenna · Dendrimer - Energy transfer · Lanthanide luminescence $\cdot$ Nanocrystals $\cdot$ Near-infrared · Metal-organic frameworks

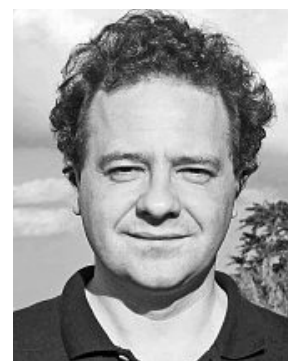

Stéphane Petoud studied chemistry at the University of Lausanne, Switzerland and received his Diploma in 1992. He obtained his $\mathrm{PhD}$ degree in 1997 from the Institute of Inorganic and Analytical Chemistry, University of Lausanne, in the area of lanthanide coordination chemistry
${ }^{\star}$ Correspondence: Prof. Dr. S. Petoud

Department of Chemistry

University of Pittsburgh

219 Parkman Ave. Pittsburgh

PA 15260, USA

Tel.: +1 4126248210

Fax: +1412 6248611

E-mail: spetoud@pitt.edu and spectroscopy under the supervision of Professors Jean-Claude G. Bünzli and Claude Piguet. From 1997 to 2000, he continued his chemistry studies in the group of Professor Kenneth N. Raymond at the University of California, Berkeley as a postdoctoral fellow with successive financial supports from the National Swiss Science Foundation, the Leenaards Foundation and from the Novartis Stiftung. From 2000 to the end of 2001, he was a staff researcher in the group of Professor Kenneth N. Raymond. In 2001, he co-founded Lumiphore Inc. with Professor Kenneth Raymond. Lumiphore (Richmond, CA, USA), is a startup company that creates and develops luminescent reporters for bioanalytical applications. Stéphane Petoud joined the Faculty of the Department of Chemistry of the University of Pittsburgh in January 2002. His research program involves the design, synthesis, analysis and use of luminescent lanthanide compounds (small molecule, macromolecules and nanomaterials) possessing controlled properties. The properties of these luminescent compounds are targeted for specific applications such as reporters and sensors for bioanalytical applications and for in vitro and in vivo biologic imaging.

\section{Introduction}

Since its establishment in 2002 in the Chemistry Department of the University of Pittsburgh, a major research direction of the Petoud group is targeted at the creation of luminescent reporters and sensors based on lanthanide cations emitting in the visible and in the near-infrared. We do fundamental research for the creation of compounds whose luminescence properties match the requirements of specific applications, with a special emphasis on bioanalytical and biological imaging applications. One 
of our global goals is to expand the field of application of luminescent lanthanide compounds by creating antennae that will allow i) the sensitization of a larger number of lanthanide cations with more diverse emission wavelengths and ii) to create novel types of reporters and sensors suitable for the detection in various types of applications including the detection of biological molecules and biological activities. To achieve this goal, a key component of the research is the design and synthesis of appropriate sensitizers for luminescent lanthanide cations emitting in the visible and in the near-infrared domains. The creation of such luminescent reporters with controlled properties includes the rationalization between structure and properties.

In this review article, we discuss several projects that we have developed for the investigation of novel antennae using different strategies. Our approach is multidisciplinary in nature and combines different fields of chemistry: analytical, inorganic, material, and biological chemistry. The combination of synthetic chemistry (molecular (organic and inorganic) and material chemistry) with analytical chemistry and spectroscopy is a specific signature of the Petoud group.

We have followed two main avenues for the creation of novel lanthanide antennae: i) small molecules of complexes and ii) polymetallic lanthanide compounds (complexes and nanocrystals).

\section{Background}

\subsection{Advantages of Luminescent Lanthanide Complexes as Report- ers for Applications}

\subsubsection{Lanthanide Complexes Are Photostable}

Most lanthanide compounds do not photobleach. ${ }^{[1]}$ Trivalent electron-deficient lanthanide cations stabilize the excited states of bound organic ligands, preventing irreversible photoreactions when the complex is irradiated. Therefore, protection from light is not required, yielding a long shelf life, easy manipulation in ambient laboratory conditions and most importantly, allows for long exposure times and repeated experiments.

\subsubsection{Lanthanide Cations Have Long Luminescence Lifetimes}

The luminescence lifetimes of lanthanide cations are in the range of micro- to milliseconds, ${ }^{[2,3]}$ which is much longer than the pico- to nanosecond lifetimes that are typical for fluorescent organic molecules and proteins. The long luminescence lifetimes allow simple and accurate discrimination of the lanthanide complex signal from autofluorescence (background

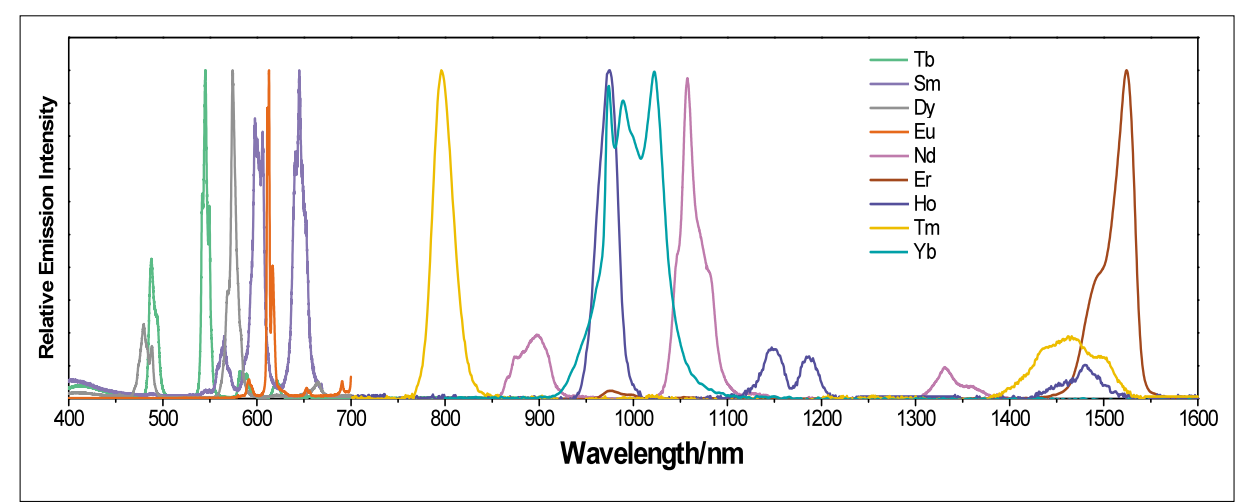

Fig. 1. Normalized emission spectra of luminescent lanthanide complexes in solution, illustrating the sharp bands and minimal overlap of lanthanide luminescence..$^{[6,7]}$ fluorescence) through time-resolved measurements, providing enhanced detection sensitivity. ${ }^{[4,5]}$

\subsubsection{Lanthanide Cations Have Unique Spectral Properties}

Due to their electronic properties, lanthanide cations can have emission bands ranging from the ultraviolet to near-infrared (NIR) regions. Since interactions between lanthanide cations and ligands are predominately electrostatic, ${ }^{[1]}$ the emission of lanthanide complexes appear as atom-like, sharp emission bands (Fig. 1).[6,7]

The energy positions of these bands also do not vary significantly with changes of experimental conditions such as temperature, pressure, $\mathrm{pH}$ or biological environments. These bandwidths are significantly more narrow than those of organic fluorophores and luminescent semiconductor nanocrystals (quantum dots). ${ }^{[8]}$ There is also a large energy gap between the absorption and emission bands of lanthanide complexes, minimizing luminescence reabsorption. Their unique properties allow lanthanide emission bands to be easily spectrally discriminated from other signals, allowing enhanced detection sensitivity in complex mixtures.

\subsubsection{Near-infrared Luminescence}

The use of NIR photons is attractive for bioanalytical and biological imaging for several reasons. Biological tissues do not have significant native NIR fluorescence, therefore use of NIR photons eliminates background fluorescence, one of the most important issues for biological imaging. NIR photons can cross deeply into tissues without causing damage or significant loss of intensity due to the low absorption of NIR photons in such media. ${ }^{9]}$ Furthermore, the use of NIR light is a promising strategy to obtain high-resolution pictures of deep tissues using optical imaging, as NIR light scatters less than visible light scattering power). ${ }^{[10]}$

\subsection{Requirements for Luminescent Lanthanide Compounds}

In order for lanthanide compounds to be sufficiently luminescent to allow (diffraction is proportional to $1 /(\lambda)^{\mathrm{SP}}, \mathrm{SP}=$

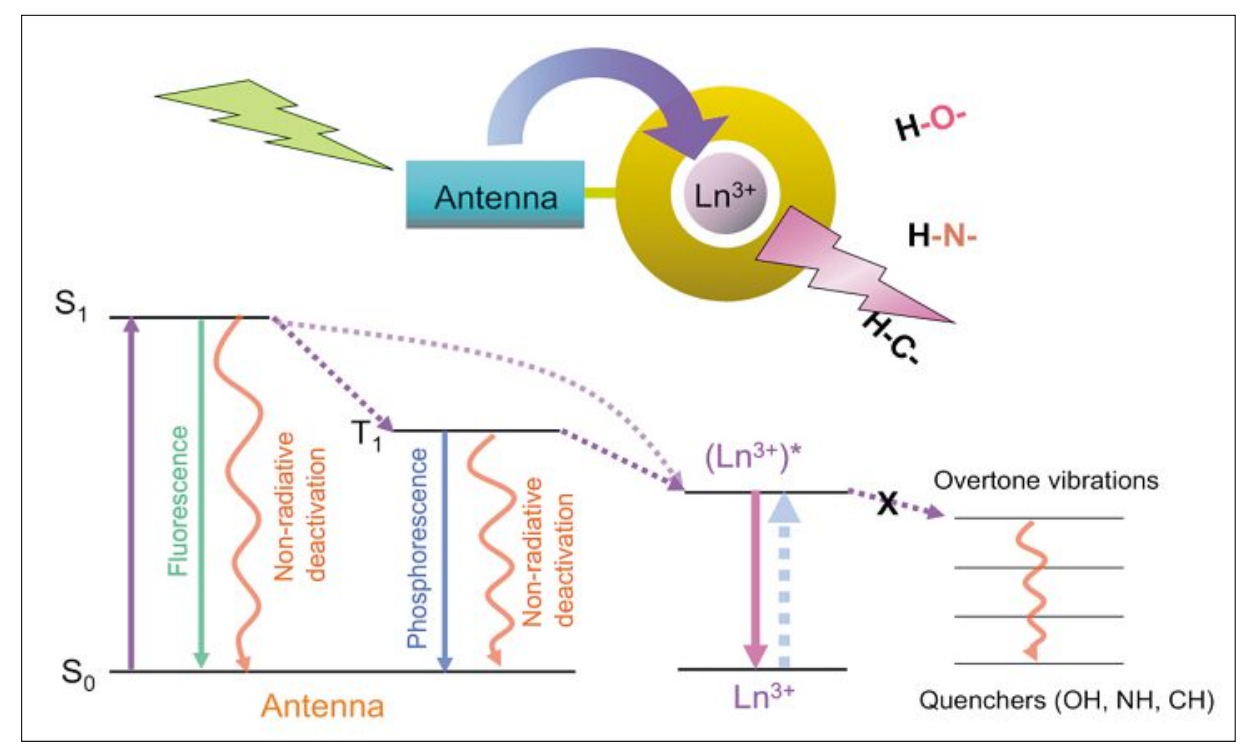

Fig. 2. Schematic representation of the antenna effect. 
good detection sensitivity, several requirements must be considered for their design. Since $\mathrm{f} \rightarrow \mathrm{f}$ transitions are forbidden by the Laporte rule, free lanthanide cations have low extinction coefficients and cannot be directly excited with good efficiency. ${ }^{[1]}$ This limitation can be overcome by placing the lanthanide cation in close proximity to a suitable sensitizer, which should absorb large quantities of excitation photons and convert the resulting energy to the lanthanide metal ion. This process is named the 'antenna effect' (Fig. 2). [11,12]

As a second requirement, in order to prevent non-radiative deactivation of the lanthanides' excited states, the cations must be protected from interactions with high energy overtones such as those of $\mathrm{C}-\mathrm{H}, \mathrm{N}-\mathrm{H}$, and $\mathrm{O}-\mathrm{H}$ vibrations from molecules in proximity. Such protection can be achieved through complete coordination of the lanthanide cation (coordination numbers are usually between 8 and 10 in solution $)^{[1,13]}$ and/or by designing ligands that sterically prevent solvent access to the metal cation. The compound must be thermodynamically stable or kinetically inert in order to prevent its dissociation in challenging conditions such as those present in solution at low concentration. This requirement is crucial to maintain the luminescence properties of the complex (the antenna must remain at close proximity to the lanthanide cation for the energy transfer to occur) and to prevent the release of lanthanide cations in biological systems since these are toxic when labile. ${ }^{[14,15]}$

\subsection{Current Limitations}

Today, despite all these advantageous properties that would be highly beneficial for a broad range of applications, the number of luminescent lanthanide compounds used in applications is relatively small, especially in the field of bioanalytical applications and biologic imaging. For a long time, it has mostly been restricted to compounds emitting in the visible domain for immunoassays on well-plate samples. ${ }^{[4,5]}$ Recently, there has been an impressive number of articles that have revealed exciting promises for the use of luminescent lanthanide compounds for cellular imagery. ${ }^{[16]}$ Nevertheless, today, the number of suitable compounds remains still limited and this situation can be partly explained by the fact that only few lanthanide compounds match the requirement for practical applications, especially the ability to emit a large number of photons per discrete unit that strongly affects the detection sensitivity. The development of NIR-emitting lanthanide complexes in both aqueous and non-aqueous media has been an increasingly active research area in recent years. Nevertheless, today there are no examples

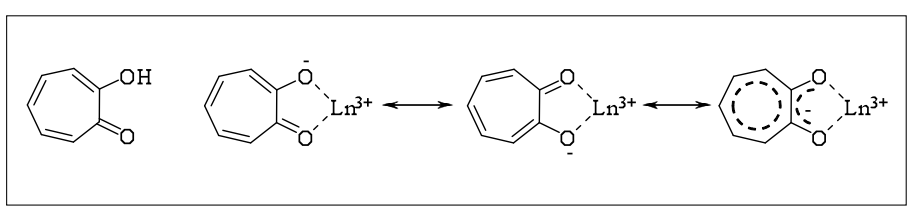

Fig. 3. Structure of tropolone and tropolonate's coordination mode with lanthanide ion.

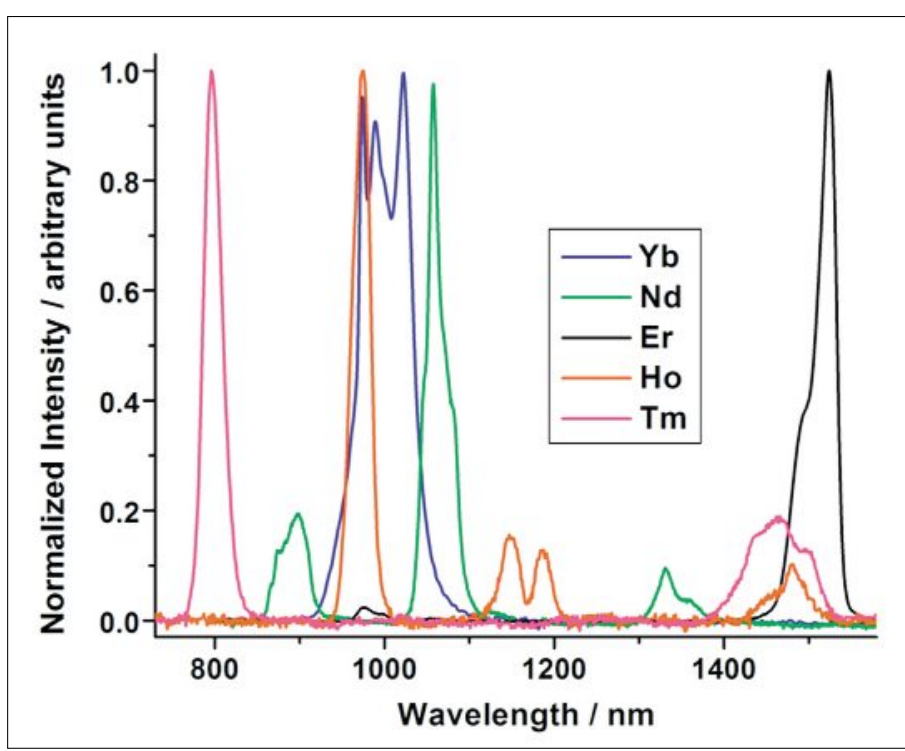

Fig. 4. Normalized emission spectra in the NIR of $\left[\mathrm{Ln}(\mathrm{L})_{4}\right]^{-}$ with $\mathrm{Ln}=\mathrm{Yb}, \mathrm{Nd}$, Er, $\mathrm{Ho}$ and $\mathrm{Tm}$ in DMSO $\left(10^{-5} \mathrm{M}\right)$ at $298 \mathrm{~K}$ upon ligand excitation at $340 \mathrm{~nm}$.

of NIR lanthanide compounds available for biologic imaging. The actual complexes formed with NIR-emitting lanthanide cations have low quantum yields in comparison to visible-emitting lanthanide compounds limiting the number of emitted photons per discrete molecule.

\subsection{Challenges}

The main challenges for lanthanide coordination and material chemists interested in their photophysical properties is to develop compounds able to emit a larger number of photons per unit of volume. This goal may be obtained not only by increasing the quantum yields (increase of the antennae-to-lanthanide energy transfer and improved protection of the lanthanide cations from quenching vibrations) but also by increasing the amount of lanthanide emitters per unit of volume (polymetallic systems) and/or by increasing the overall absorption of each discrete molecule.

\section{Monometallic Complexes}

The strategy to develop monometallic complexes as luminescent lanthanide compounds has been triggered by the idea to synthesize species that are easy to characterize. It is also attractive to work with small molecules since it easier to analyze the relationship between the electronic structure of the antenna and the photophysical properties of the resulting compounds.

\subsection{Tropolonates and Ligands Derived from Tropolonates}

Tropolonate is a seven-member aromatic ring which functions as a bidentate chromophoric sensitizer (Fig. 3).

We have successfully demonstrated that this molecule is able to coordinate and to sensitize several lanthanide cations. We have structurally characterized a large number of structures obtained with these chromophores $\left(\mathrm{Ln}^{3+}=\mathrm{Tb}^{3+}, \mathrm{Dy}^{3+}, \mathrm{Ho}^{3+}\right.$, $\mathrm{Er}^{3+}, \mathrm{Tm}^{3+}, \mathrm{Yb}^{3+}$ and $\left.\mathrm{Lu}^{3+}\right)$. In all cases, we have identified the $\left[\mathrm{Ln}(\mathrm{L})_{4}\right]^{-}\left(\mathrm{L}=\mathrm{C}_{7} \mathrm{H}_{5} \mathrm{O}_{2}\right.$; tropolonate) type of complex formed by reaction of four deprotonated tropolonate ligands with one equivalent of lanthanide cation. ${ }^{[7,17]}$ Remarkably, we have identified that tropolonate is efficient enough to sensitize five different NIR-emitting lanthanide cations: $\mathrm{Nd}^{3+}, \mathrm{Er}^{3+}, \mathrm{Ho}^{3+}, \mathrm{Tm}^{3+}$, and $\mathrm{Yb}^{3+}$ in solution (Fig. 4). Quantum yields of the complexes formed with $\mathrm{Yb}^{3+}$ $\left(1.9 \cdot 10^{-2}\right.$ in DMSO) are comparable to the highest reported quantum yield values for other lanthanide complexes that emit in the NIR domain in organic solvents.

The limitation of this system is that the structure in the solid state indicates that the lanthanide cation has a coordination number of 8 provided by the four tropolonate ligands. The situation is different in solution, the coordination being 9, the additional coordination site being occupied by a molecule of solvent. The presence of this molecule of solvent induces a strong nonradiative deactivation of the luminescence of the lanthanide cation, especially when the solvent is water. As a quantitative result, 


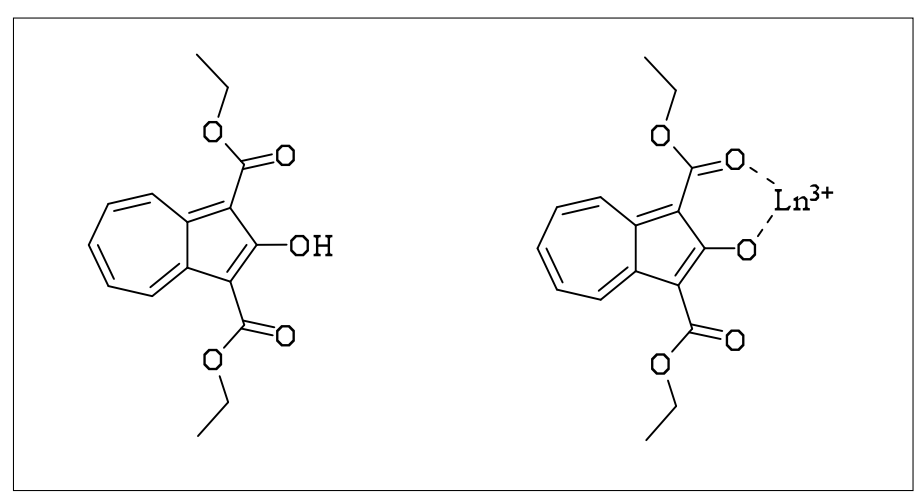

Fig. 5. Molecular structure of $\mathrm{HAz}$ and the proposed bidentate coordination mode of $\mathrm{Az}^{-}$with $\mathrm{Ln}^{3+}$.

Fig. 6. Normalized excitation (dash) and emission spectra (plain) of four $\left[\mathrm{Ln}(\mathrm{Az})_{4}\right]^{-}$ complexes $\left(10^{-5} \mathrm{M}\right.$ in $\left.\mathrm{CH}_{3} \mathrm{CN}, \lambda_{\mathrm{ex}}=380 \mathrm{~nm}\right)$.

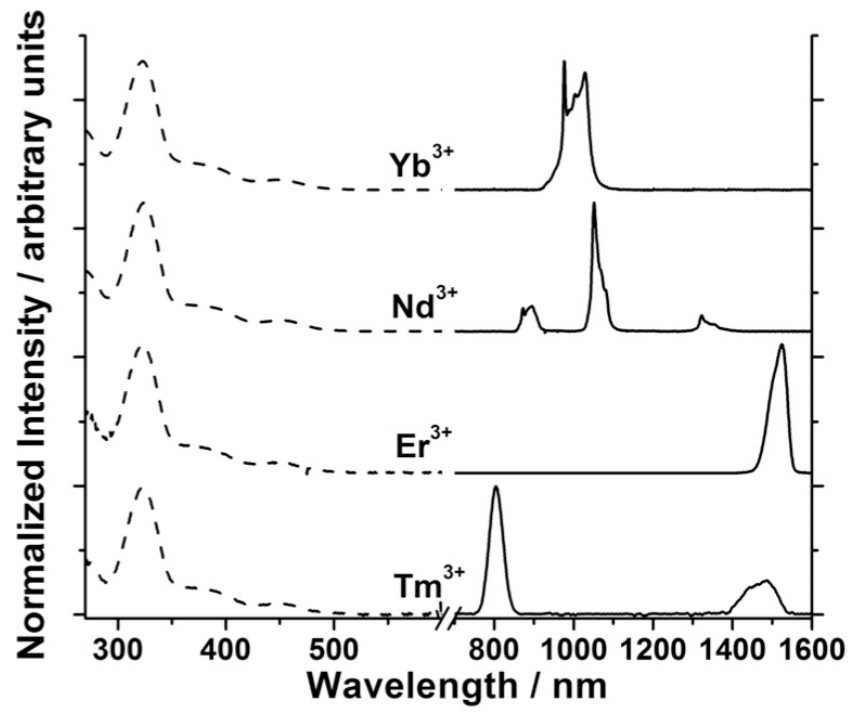

the quantum yield value of the $\left[\mathrm{Yb}(\mathrm{L})_{4}\right]$ complex recorded in water is almost two orders of magnitude smaller that the value observed in DMSO. This limitation is expected to be removed in the future by attaching the four tropolonate sensitizing moieties to a common backbone which should improve the protection of the lanthanide cation against non-radiative deactivation.

\subsection{Complexes Formed with Derivatives of Azulene}

Another family of ligands that has been studied incorporates the azulene moiety (Fig. 5) as a novel sensitizer, which shares some structural similarities with tropolonate (incorporation of a seven-member ring moiety). [18]

It has been chosen on the criterion of its triplet state located at lower energy, on the hypothesis of a better match with the accepting levels of several NIR-emitting lanthanide cations. The $\left[\mathrm{Ln}(\mathrm{Az})_{4}\right]^{-}$lanthanide complexes formed with the bidentate azulene ligand $\mathrm{HAz}$ were synthesized, and their photophysical properties analyzed. This ligand-chromophore system provides an efficient sensitization for four different NIR-emitting lanthanide cations: $\mathrm{Yb}^{3+}$, $\mathrm{Nd}^{3+}, \mathrm{Er}^{3+}$ and $\mathrm{Tm}^{3+}$ (Fig. 6).
The quantum yields of the $\mathrm{Nd}^{3+}$ and $\mathrm{Yb}^{3+}$ complexes $\left(3.7 \cdot 10^{-3}\right.$ and $3.2 \cdot 10^{-2}$ respectively, both in $\left.\mathrm{CH}_{3} \mathrm{CN}\right)^{[18]}$ are among the highest values reported for NIR-emitting lanthanide complexes in solution, which we attribute to a combination of efficient ligand-to-lanthanide energy transfer and good protection of the lanthanide cation from non-radiative deactivations. The fact that the azulene-based ligand can sensitize not only $\mathrm{Yb}^{3+}, \mathrm{Nd}^{3+}$, and $\mathrm{Er}^{3+}$, but also $\mathrm{Tm}^{3+}$ indicates that it is a promising sensitizer for NIR lanthanide ions, potentially suitable for biological imaging. These derivatives of azulene are another novel type of sensitizer suitable for several NIR-emitting lanthanides, including $\mathrm{Tm}^{3+}$ whose emission wavelength is particularly interesting for biological imaging (minimal absorption of tissues and biological fluids). These results validate our hypothesis of sensitizers with triplet states located at lower energy being favorable for NIR-emitting lanthanide cations. The four bidentate ligands coordinated to the central lanthanide cation in the $\mathrm{ML}_{4}$ complex provide good protection of the lanthanide cations against non-radiative deactivations, an improvement over the complexes formed with the tropolonate ligands.

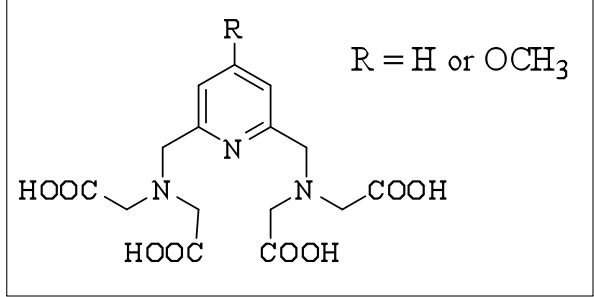

Fig. 7. Structure of the ligands.

\subsection{Lanthanide Complexes for Bimodal MRI and NIR Lumines- cence Detection}

Bimodal magnetic and optical imaging agents are in high demand for biological imaging, combining the resolution of one of these techniques with the sensitivity of the other. Complexes based on lanthanide cations have electronic structures that can provide the suitable properties for both required magnetic and optical properties. In collaboration with Dr. Eva Tóth (Centre de Biophysique Moléculaire, CNRS, Orléans, France) and Dr. Franck Suzenet (Institut de Chimie Organique et Analytique, Université d'Orléans, France), we have created a versatile scaffold for $\mathrm{Ln}^{3+}$ complexation (Fig. 7) where MRI and luminescence requirements are simultaneously satisfied using a common ligand.[19]

At this proof of principle stage of the project, we have demonstrated that even bishydrated NIR-emitting $\mathrm{Nd}^{3+}$ complexes can emit a significant signal in aqueous solution the pyridyl derivative unit acting as the antenna (Fig 8).

These complexes have a quantum yield $\left(9.7 \cdot 10^{-5}\right.$ for the $\mathrm{Nd}^{3+}$ complex in $\left.\mathrm{H}_{2} \mathrm{O}\right)^{[19]}$ in the same range as those of the most optimized chelates described so far in the literature. ${ }^{[20]}$ These chelates are remarkably inert, related to the rigidity of the pyridyl skeleton. The analysis of the photophysical properties of the $\mathrm{Nd}^{3+}$ complexes formed with the ligands of this family indicate that the pyridyl synthon is a prime candidate for the development of bimodal MRI/NIR imaging probes. In the perspective of biological applications, we are currently modifying the ligand structure to displace the excitation wavelength towards lower energies where tissues and blood have lower absorbance to i) prevent damage to the biological sample and ii) to allow deeper tissue penetration of the photons for non-invasive optical diagnostics. The lowering of the energies of the triplet states is also expected to improve the sensitizer-to-lanthanide energy transfer as demonstrated from our previous work based on azulene. These structural modifications of the ligand, however, are not expected to influence the bishydrated nature and kinetic inertness of the complexes. 


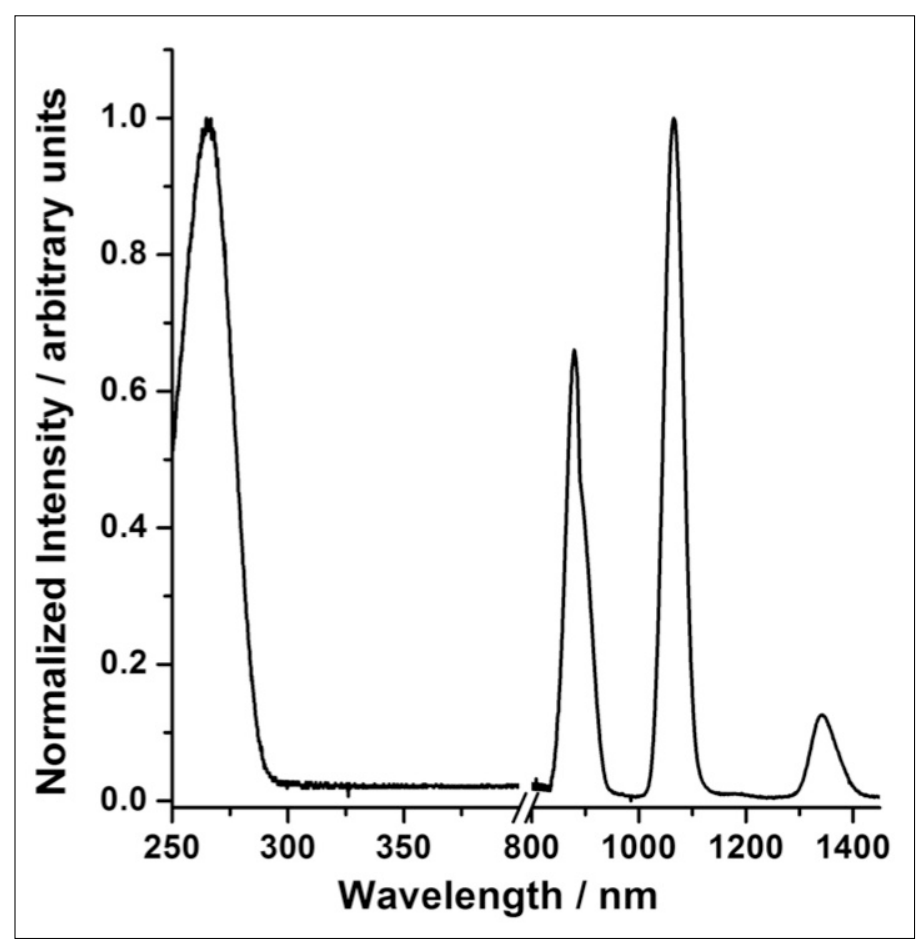

Fig. 8. Normalized excitation (dots, $\lambda_{\text {em }}=1065 \mathrm{~nm}$ ) and emission $\left(\lambda_{\text {ex }}=\right.$ $267 \mathrm{~nm}$ ) spectra of the NdL complex formed with ligand $\mathrm{R}=\mathrm{H} .5 .1 \times 10^{-4}$ $\mathrm{M}$ in HEPES buffer $0.01 \mathrm{M}, \mathrm{pH}=7.02$, ionic strength $\mathrm{KCl} 0.01 \mathrm{M}$.

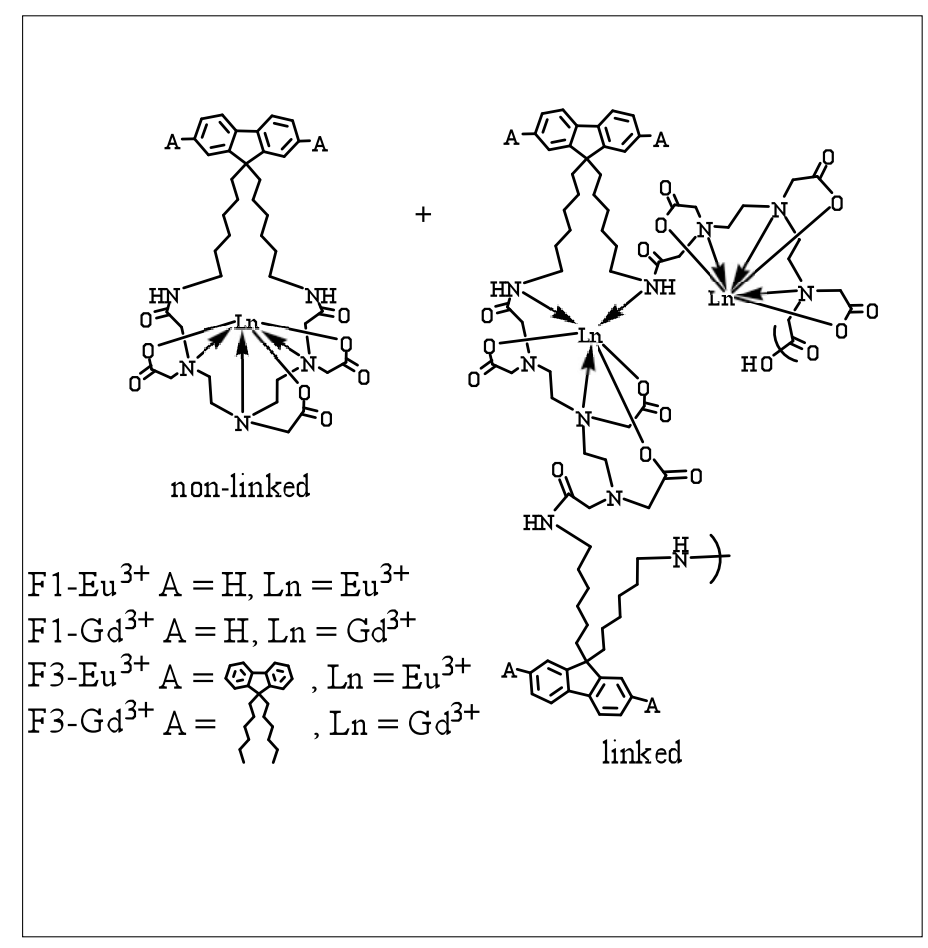

Fig. 9. Molecular structures of the complexes.
In summary, this prototype lanthanide complex is the first report of a lanthanidebased bimodal agent that combines MRI activity with NIR luminescence. We have demonstrated for the first time with this system that the presence of two $\mathrm{H}_{2} \mathrm{O}$ molecules bound to the $\mathrm{Ln}^{3+}$, beneficial for MRI applications of the $\mathrm{Gd}^{3+}$ analogue, is not an absolute limitation for the development of NIR luminescent agents; there exist feasible routes towards $\mathrm{Ln}^{3+}$-based, bimodal probes suitable for both MRI and NIR optical imaging.

\subsection{Fluorene Oligomers as Versatile Antenna for Luminescent Lantha- nide Cations}

As another type of antenna for luminescent lanthanide cations, we have recently tested the use of derivatives of mono- and terfluorene oligomeric groups as sensitizer for the $\mathrm{Eu}^{3+}$ cation in collaboration with my colleague Prof. Tara Y. Meyer and Dr. Harry M. Edenborn at the National Energy Technology Laboratory, U.S. Department of Energy in Pittsburgh. The rationale for testing this oligomeric approach is based on the hypothesis that the number of oligomeric units can control the energy of the triplet state of the antenna and the resulting sensitizer to lanthanide energy transfer. This strategy is expected to allow for easier tuning of the sensitizer-to-lanthanide energy transfer for different lanthanide cations by controlling the match of the energies between the donor's electronic levels and the accepting levels of the lanthanide cations without having to recreate a whole new molecular system for the coordination and sensitization of the lanthanide cations. To achieve this goal, we have designed sensitizing units where the chromophore is not bound directly to the lanthanide cation but is maintained at a controlled distance using different building blocks that include an antenna, a lanthanide ligand, a linker and a lanthanide cation (Fig. 9). This strategy allows the facile modification of the sensitizing oligomeric structure for the tuning of intramolecular energy transfer without affecting the other properties of the complex.

In preliminary experiments, ${ }^{[21]}$ we have demonstrated that using this strategy, we have been able to obtain a promising antenna effect for the visible-emitting $\mathrm{Eu}^{3+}$ using new ligands incorporating mono- and terfluorene sensitizers. The performance of the two types of fluorene complexes established a proof of principle that oligomer length can be used to tune the sensitizer-to-lanthanide energy transfer. The luminescence lifetimes recorded on $\mathrm{Eu}^{3+}$ for both types of $\mathrm{Eu}^{3+}$ complexes incorporating derivatives of mono and terfluorene sensitizing units were fairly similar as an indication that, with this approach, we can change the sensitizing unit without affecting the environment around the $\mathrm{Eu}^{3+}$ cation and that this cation is well protected from the en- vironment. The quantum yield of the $\mathrm{Eu}^{3+}$ complex formed with the unit carrying the terfluorene antenna recorded upon $\mathrm{Eu}^{3+}$ monitoring $\left(6.7 \cdot 10^{-2} \text { in DMSO }\right)^{[21]}$ can be considered as high in comparison to commercially available complexes despite the incomplete energy transfer. These results suggest that this versatile modular system has significant potential for future applications since it allows relatively easy modification of the chromophoric units without having to re-engineer the whole system when a change of the electronic structure is required.

\section{Polymetallic Complexes}

As discussed previously, in many cases, a limitation exists with the photon output of monometallic lanthanide complexes preventing their use for a broad range of applications, especially those emitting in the NIR. We want to improve this situation by better understanding the parameters that control the intramolecular energy transfer of our systems and by improving the protection of the lanthanide cations against non-radiative deactivations.

In order to take immediate advantage of the properties of lanthanide complexes for practical applications, we have decided to explore a novel strategy where the number of photons per unit of molecular volume is maximized by the creation of polymetallic lanthanide emitters having very large 


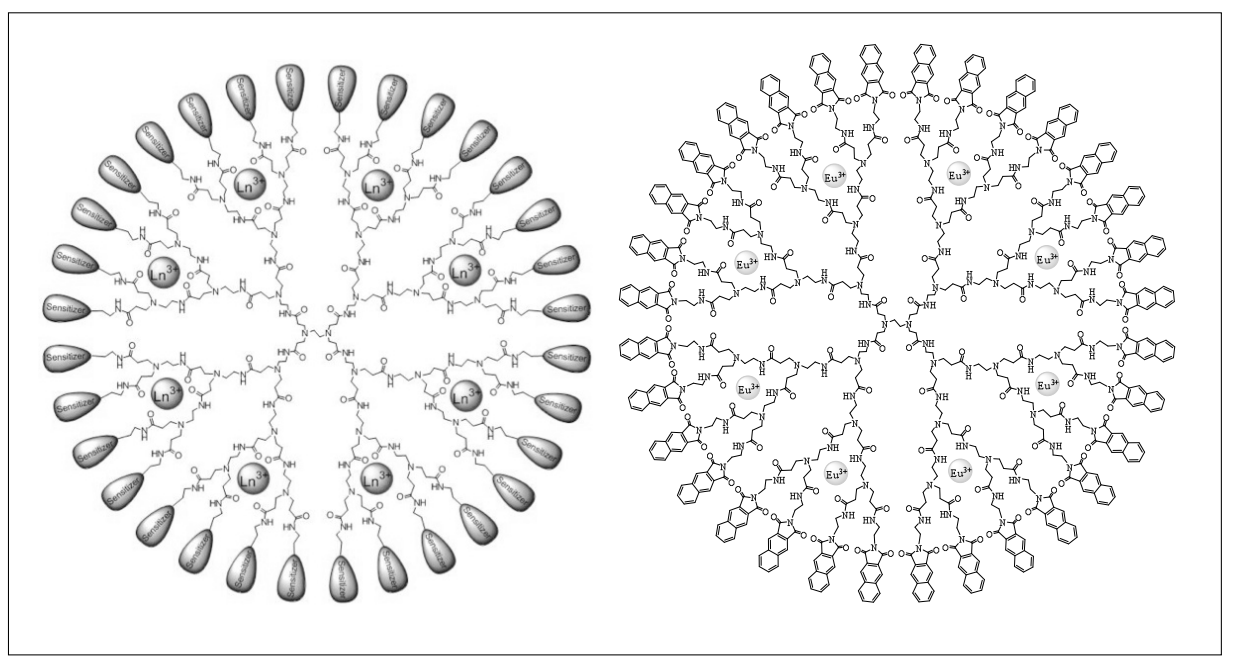

Fig. 10. Left: general schematic structure of a dendrimer ligand formed with a generation-3 PAMAM dendrimer. The ends of the branches are substituted by sensitizers. Right: polymetallic complex formed by reaction of a generation-3 PAMAM dendrimer whose branches have been functionalized with 32 2,3-naphthalimide groups with $8 \mathrm{Eu}^{3+}$ cations.

absorption. ${ }^{[22]}$ A larger number of photons per unit of molecular volume will induce a better detection sensitivity. We took into account the fact that the luminescence intensity of a lanthanide compound is proportional to i) the quantum yield (which reflects the efficiency of intramolecular energy transfer and protection of the lanthanide cation from non-radiative deactivation, currently a limiting factor) ii) the number of lanthanide cations per unit of volume and iii) the molar absorptivity of the compound. To test this strategy, we have tested three different families of chemical species for the development of more luminescent lanthanide compounds: a) macrocyclic polymetallic dendrimer complexes and b) lanthanide-containing nanocrystals (semiconductor and fluoride) and c) metal-organic frameworks containing lanthanides.

\subsection{Polymetallic Dendrimer Complexes}

We have initiated a project targeted at the synthesis, study and use of luminescent dendrimer lanthanide complexes that have the advantage of forming polymetallic lanthanide compounds. The lanthanide cations are coordinated to the amide oxygen atoms present on the internal branches of dendrimers based on poly(amidoamine) (PAMAM) (Fig. 10, left).

Lanthanide sensitizers are substituted on the branch termini of these dendrimers, allowing a large number of chromophores to be attached on each discrete dendrimer molecule. This approach will help with the maximization of the absorption of the compound, an advantage to increase the number of photons emitting per each individual molecule (in addition to the polymetallic advantage). This design also allows the choice of a broader variety of lanthanide sensitizer since, unlike the bidentate molecule systems we have been using for the formation of small molecule type of complexes, the chromophoric group does not need to possess atoms or groups that will form bonds with lanthanides.

To establish a proof of principle of the validity of this conceptual approach, we have synthesized a ligand based on a generation-3 PAMAM dendrimer functionalized with 32 2,3-naphthalimide chromophoric groups on the end-branches (Fig. 10, right). [23] The choice of the 2,3-naphthalamide group was based on the high population of its triplet state due to the particularly efficient intersystem crossing.[24] With this ligand system, we have demonstrated that we can identify the formation of polymetallic $\mathrm{Eu}^{3+}$ complexes using luminescence titrations. The formation of a species corresponding to a dendrimer:lanthanide ratio of 1:8 was determined on the criteria of the strongest $\mathrm{Eu}^{3+}$ emission signal per lanthanide cation (as a result of the energy transfer and more efficient protection of the lanthanide cation against non-radiative deactivations). It took at least seven days for the complete formation of the complex, suggesting kinetic inertness. Luminescence lifetimes indicate that all eight cations are well protected from non-radiative deactivation by the dendritic structure. Interestingly, the observation of a single luminescence decay value for all eight lanthanide cations coordinated in the dendrimer is a strong indication that each of the eight cations in the dendrimer adopts a similar coordination environment. Despite the limited efficiency of the sensitization of $\mathrm{Eu}^{3+}$, the absolute quantum yield being $0.06 \%,[23]$ the presence of eight lanthanide cations bound in the dendrimer structure (polymetallic advantage), combined with good protection of the luminescent lantha- nide cations from the environment and high absorption resulting from the presence of 32 naphthalimide groups lead to the emission of a fairly intense red signal arising from the $\mathrm{Eu}^{3+}$ cations. This emission light is easily visible in solution with the naked eye under irradiation with $354 \mathrm{~nm}$ UV light.

\subsection{Lanthanide-containing CdSe Semiconductor Nanocrystals}

We have also tested a complementary and novel strategy that combines i) the formation of polymetallic lanthanide compounds with a high density of lanthanide cations per unit of volume, ii) high extinction coefficients and iii) the optimized protection of the lanthanide cations against non-radiative deactivation through the structure of nanocrystals. In comparison to the approach based on dendrimers that has been presented previously, the use of nanocrystals potentially allows a larger density of lanthanide cations per unit of volume and therefore an increased number of photons. Since nanocrystals only possess low energy vibrations (network vibrations), the excited states of the lanthanide cations will not be quenched by the nanocrystal structure in addition to getting maximal protection from the environment. The conceptual idea at the level of the antenna for this system is to use the electronic structure of the semiconductor nanocrystals to sensitize the lanthanide cations located in the core and/or at the surface of the nanocrystals. The energies of the electronic donating levels of the nanocrystals are controlled by the size of these nanocrystals, allowing the potential tuning of the energy transfer to the different lanthanide cations by the synthetic conditions. Semiconductor nanocrystals such as CdSe have very high extinction coefficients, an additional advantage to maximize the number of photons emitted by each discrete nanocrystal. We have developed syntheses that allow the incorporation of the lanthanide cations within the nanocrystals that have a size that does not exceed 2-4 nm. Small sizes are important for these luminescent reporters to be used at a later stage in biological applications (minimization of the interference between the nanocrystals and the biological system to study). We have demonstrated for the first time that $\mathrm{CdSe}$ can serve as an antenna for $\mathrm{Tb}^{3+}$ in lanthanide-doped CdSe:Tb nanocrystals (Fig. 11).[25]

We have also demonstrated that the structure of these nanocrystals protects extremely well the lanthanide cations against non-radiative deactivations. The presence of two independent luminescence lifetime values is a good indication that the lanthanide cations are located both at the surface and in the core of the nanocrystals. 

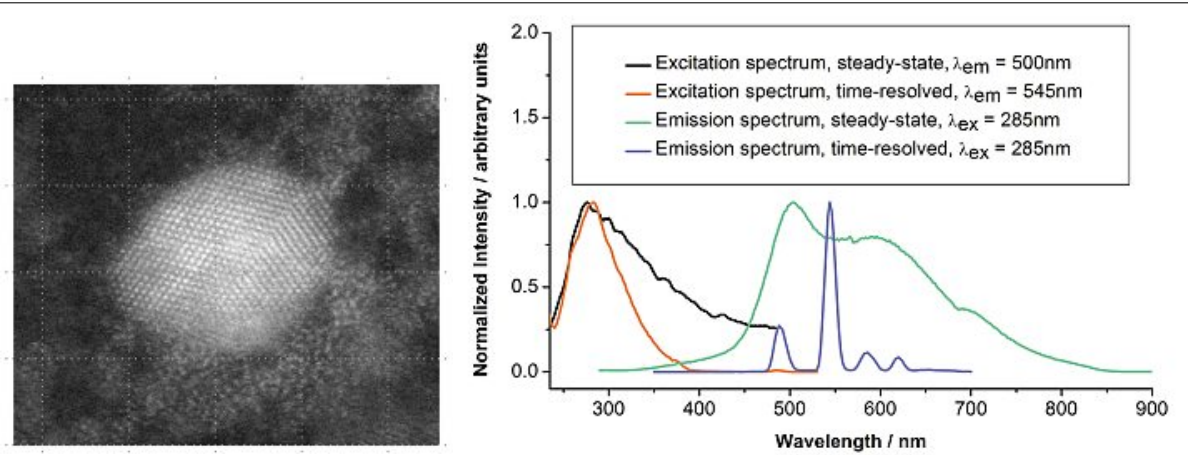

Fig. 11. Left: Image of a Tb ${ }^{3+}$ doped CdSe:Tb nanocrystals obtained from high resolution transmission electron microscopy experiment. The diameter of the nanocrystals is approximately $2.8 \mathrm{~nm}$. Right: normalized steady-state and time-resolved excitation and emission spectra of $\mathrm{Tb}^{3+}$ doped CdSe:Tb nanocrystals in chloroform. The $\mathrm{Tb}^{3+}$ emission is in the same range as the nanocrystal emission, however, it is easily distinguished through time-resolved measurements. These spectra demonstrate that energy transfer through the nanocrystals to the lanthanide cation is possible.

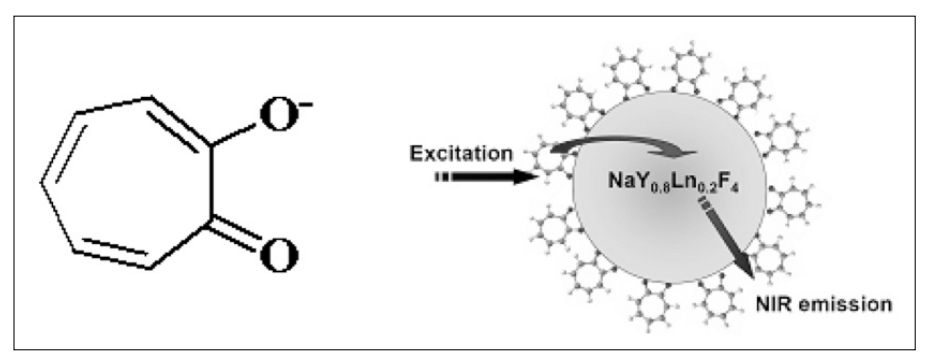

Fig. 12. Left: tropolonate sensitizer and right: schematic illustration of tropolonate capped $\mathrm{Nd}^{3+}$ or $\mathrm{Yb}^{3+}$ doped $\mathrm{NaYF}_{4}$ nanocrystals.

\subsection{Organic Tropolonate Ligands for the Sensitization of Polymetallic Ln $^{3+}$-Doped $\mathrm{NaYF}_{4}$ Nanocrystals}

We have tested another sensitization strategy that combines the use of an organic chromophore for the sensitization of the luminescent lanthanide and nanocrystal for the formation of polymetallic species where the luminescent cations are well protected from the environment (Fig. 12).

Several organic antennae are located at the surface of lanthanide-doped yttrium fluoride nanocrystals. This approach is appealing since it takes advantage of a broad choice of organic sensitizers. The lanthanide cations are well protected within the structure of the nanocrystal from non-radiative deactivations, which is especially appealing for NIR-emitting lanthanides that are easily quenched due to the small gap between their excited and ground states.

We have tested this sensitization/protection strategy with two different NIRemitting lanthanide cations, $\mathrm{Nd}^{3+}$ and $\mathrm{Yb}^{3+}$, using the tropolonate sensitizer that we have used previously (see Section 3.1) ${ }^{[7]}$ for the formation of molecular $\mathrm{ML}_{4}$ complexes.[26] Combining protection against non-radiative deactivations, provided by the inorganic matrix of $\mathrm{NaYF}_{4}$ nanocrystals with sensitization from tropolonate ligands capped on their surface, the lanthanide cation-centered luminescence was observed through the ligand excitation and demonstrates that tropolonate ligands can provide an antenna effect. The extended lanthanide luminescence lifetimes indicate the success of this strategy aimed at protecting the lanthanide cations against nonradiative deactivations. This strategy can be potentially extended to a broader range of antenna for the optimized energy transfer. These antennae do not need to have specific binding groups for the lanthanide cations. The next steps for this work is to rationalize the effect of number of tropolonate ligands located on the surface, and the number of lanthanides, on the overall quantum yields of the compounds.

\subsection{Metal-organic Frameworks for NIR-emitting Polymetallic Lantha- nide Complexes}

In collaboration with my colleague Professor Nathaniel Rosi, we have tested an approach based on the use of metalorganic frameworks (MOFs) as a strategy to coordinate and sensitize NIR-emitting lanthanide cations. This approach is another exciting example of the strategy for the maximization of the number of lanthanide cations and number of chromophores to maximize the number of photons emitted per unit of volume. The MOF structure induces a high level of order and organization, which is appealing for the control of the luminescence properties of such compounds. MOFs are a relatively new class of porous materials consisting of metals or clusters of metals linked together with rigid organic molecules into 1-, 2-, or 3-D networks. We have tested an approach to luminescent lanthanide materials where the cations are incorporated into MOFs as the metal component of networks formed with chromophoric organic groups. The organic groups sensitize lanthanide luminescence, while the MOF framework protects lanthanide cations from quenching. These rigid architectures provide the ability to control the environment around the lanthanide cations, achieving specific coordination geometries for the cations and also allowing control over the organic components' arrangement in space, enabling in-

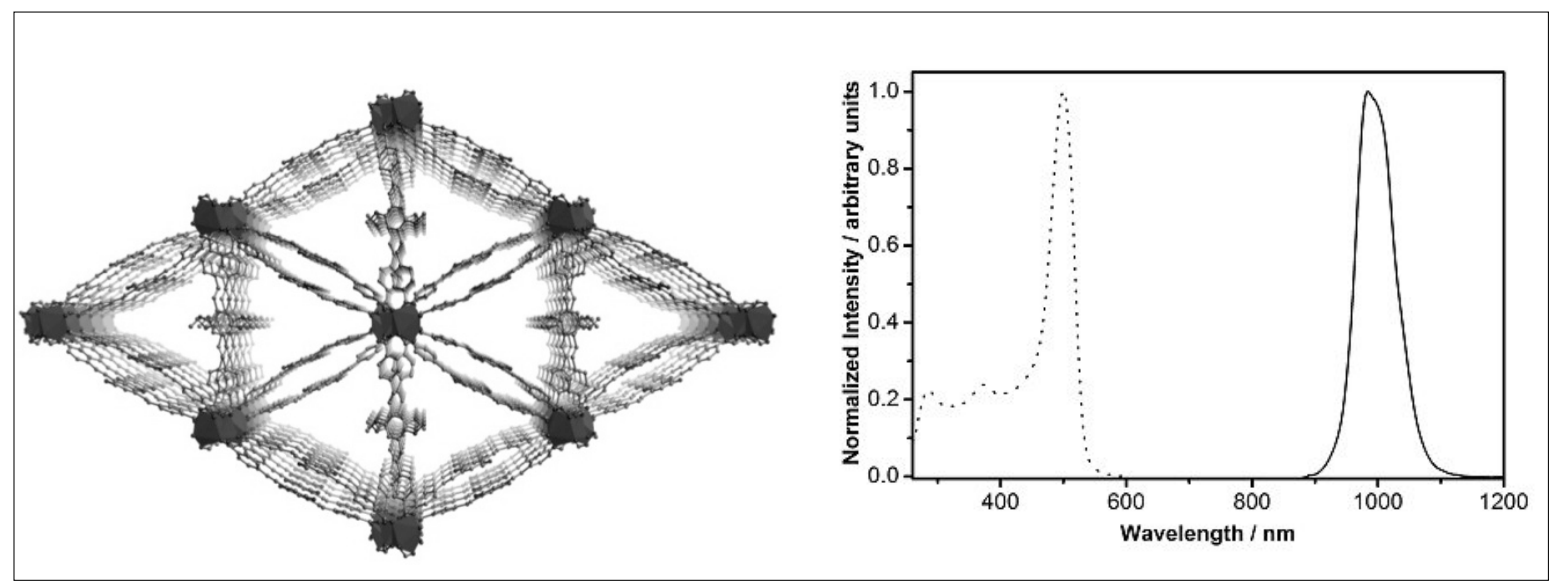

Fig. 13. Left: structural depiction of one of two MOFs formed with $\mathrm{Yb}^{3+}$ and a phenylene moiety. Right: luminescence data of the excitation (dotted; $\lambda_{\text {em }}=980$ $\mathrm{nm}$ ) and emission profiles (solid; $\lambda_{\mathrm{ex}}$ $=500 \mathrm{~nm}$ ). MOFs as crystalline solids under chloroform. 
teractions between chromophoric groups that possess lower excitation energy ranges. Two unique MOF structures have been formed with $\mathrm{Yb}^{3+}$ and a phenylene moiety (Fig. 13). [27]

Their photophysical properties were investigated with visible and NIR fluorescence spectroscopy. The MOFs display $\mathrm{Yb}^{3+}$ centered luminescence with red-shifted excitation bands, long luminescence lifetimes, and quantum yields $\left(1.8 \cdot 10^{-2}\right.$ for Yb-PVDC-2) ${ }^{[27]}$ that are among the highest reported values for NIR-emitting lanthanide compounds. The results of these preliminary studies have been recently published. ${ }^{[27]}$ In summary, we have created here another alternative strategy to sensitize and protect luminescent lanthanide cations, where the lanthanide is rigidly well-organized three-dimensionally, an appealing advantage for rational design. Despite the presence of the organic sensitizers, lanthanide cations do not appear to be strongly quenched by the vibrations of the solvent or of the chromophore. The presence of internal cavities is appealing for the design of sensors.

\section{Conclusion}

Through this review article describing different efforts by our research group for the creation and study of novel antennae to sensitize lanthanide cations emitting in the visible and in the NIR, we have demonstrated that much synthetic and spectroscopic work must be done in order to generate lanthanide compounds with novel or improved properties that can fit the requirements of a broad range of applications. Such work combines synthetic chemistry (organic, inorganic and material) with analytical chemistry and spectroscopy, producing exciting results. This effort is especially important in the field of the sensitization of NIR-emitting lanthanide cations. More specifically, there is a strong need for such complexes to be optimized to emit a larger number of photons per unit of volume for enhanced detection sensitivity. It is also important to maximize the quantum yields of these compounds by maximizing the efficiency of the intramolecular energy transfer and/or by ensuring good protection of the lanthanide cations against non-radiative deactivations. This work includes the identification and quantification of different parameters that control the energy transfer and quenching in lanthanide compounds.

We have also demonstrated through various examples the validity of our strategy where detection sensitivity is enhanced by the creation of polymetallic compounds possessing high absorbtivities in an effort to maximize the number of photons emitted per unit of volume.

There is still a significant amount of effort to be done in order to achieve these goals of being able to design lanthanide compounds with controlled photonic and magnetic properties.

\section{Acknowledgements}

Funding (complete or partial) for the different projects presented in this review article was provided through the University of Pittsburgh, through the National Science Foundation (award DBI0352346), through the National Institutes of Health (awards 1R01HL081421-01 and 1R21CA133553-01), the National Energy Technology Laboratory's on-going research in clean coal technology under the RDS contract DE-AC26-04NT41817. S.P. gratefully acknowledges Le Studium (Agency for research and international hosting associate researchers in 'Region Centre'), Orléans, France, for financial support.

Received: June 5, 2009

[1] J.-C. G. Bünzli, C. Piguet, Chem. Soc. Rev. 2005, 34, 1048.

[2] W. D. Horrocks Jr., D. R. Sudnick, Acc. Chem. Res. 1981, 14, 384.

[3] A. Beeby, I. M. Clarkson, R. S. Dickins, S. Faulkner, D. Parker, L. Royle, A. S. de Sousa, J. A. G. Williams, M. Woods, J. Chem. Soc., Perkin Trans. 2 1999, 493.

[4] a) G. Mathis, J. Biomol. Screening 1999, 4 , 309; b) E. G. Moore, A. P. S. Samuel, K. N. Raymond, Acc. Chem. Res., 2009, 42, 542.

[5] I. Hemmila, V. M. Mukkala, Crit. Rev. Clin. Lab. Sci. 2001, 38, 441.

[6] S. Petoud, S. M. Cohen, J.-C. G. Bünzli, K. N. Raymond, J. Am. Chem. Soc. 2003, 125, 13324

[7] J. Zhang, P. D. Badger, S. J. Geib, S. Petoud, Angew. Chem. Int. Ed. 2005, 44, 2508.

[8] M. Bruchez, M. Moronne, P. Gin, S. Weiss, A. P. Alivisatos, Science 1998, 281, 2013.

[9] R. Weissleder, V. Ntziachristos, Nat. Med. 2003, 9, 123.

[10] S. Kim, Y. T. Lim, E. G. Soltesz, A. M. De Grand, J. Lee, A. Nakayama, J. A. Parker, J. T. Mihaljevic, R. G. Laurence, D. M. Dor, L. H. Cohn, M. G. Bawendi, J. V Frangioni, Nat. Biotechnol. 2004, 22, 93.

[11] S. I. Weissman, J. Chem. Phys. 1942, 10, 214.

[12] N. Sabbatini, M. Guardigli, J.-M. Lehn, Coord. Chem. Rev. 1993, 123, 201.

[13] D. Parker, R. S. Dickins, H. Puschmann, C. Crossland, J. A. K. Howard, Chem. Rev. 2002 102, 1977.
[14] S. Hirano, K. T Suzuki, Environ. Health. Perspect. 1996, 104, 85.

[15] A. S. Boyd, J. A. Zic, J. L. Abraham, J. Am. Acad. Dermatol. 2007, 56, 27.

[16] a) J.-C. G. Bünzli, Chem. Lett. 2009, 38, 104; b) C. P. Montgomery, B. S. Murray, E. J. New, R. Pal, D. Parker, Acc. Chem. Res. 2009, 42, 925 ; c) J.-C. G. Bünzli, A.-S. Chauvin, C. D. B. Vandevyver, S. Bo, S. Comby, Ann. N.Y. Acad. Sci. 2008, 1130, 97; d) A. Picot, A. d'Aléo, P. L. Baldeck, A. Grichine, A. Duperray, C. Andraud, O. Maury, J. Am. Chem. Soc. 2008, 130, 1532; e) S. Faulkner, S. J. A. Pope, B. P. Burton-Pye, Appl. Spectrosc. Rev. 2005, 40, 1; f) A. Beeby, S. W. Botchway, I. M. Clarkson, S. Faulkner, A. W. Parker, D. Parker, J. A. G. Williams, J. Photochem. Photobiol. B: Biol. 2000, 57, 83; g) L. J. Charbonnière, N. Hildebrandt, R. F. Ziessel, H. G. Loehmannsroeben, J. Am. Chem. Soc. 2006, 128, 12800; h) K. Hanaoka, K. Kikuchi, S. Kobayashi, T. Nagano, J. Am. Chem. Soc. 2007, 129, 13502; i) G. Vereb, E. Jares-Erijman, P. R. Selvin, T. M. Jovin, Biophys. J. 1998, 74, 2210; j) R. C. Leif, L. M. Vallarino, M. C. Becker, S. Yang, Cytometry A. 2006, 69, 767; k) D. J. Bornhop, J. M. M. Griffin, T. S. Goebel, M. R. Sudduth, B. Bell, M. Mohamed, Appl. Spectrosc. 2003, 57, 1216; 1) M. H. V. Werts, N. Nerambourg, D. Pélégry, Y. le Grand, M. Blanchard-Desce, Photochem. Photobiol. 2005, 4, 531.

[17] J. Zhang, P. D. Badger, S. J. Geib, S. Petoud, Inorg. Chem. 2007, 46, 6473.

[18] J. Zhang, S. Petoud, Chem.-Eur. J. 2008, 14, 1264.

[19] L. Pellegatti, J. Zhang, B. Drahos, S. Villette, F. Suzenet, G. Guillaumet, S. Petoud, E. Tóth, Chem. Commun. 2008, 6591.

[20] S. Comby, D. Imbert, C. Vandevyver, J.-C.G. Bünzli, Chem.-Eur. J. 2007, 13, 936.

[21] D. S. Oxley, R. W. Walters, J. E. Copenhafer, T. Y. Meyer, S. Petoud, H. M. Edenborn, Inorg. Chem. 2009, 48, 6332.

[22] D. A. Chengelis, A. M. Yingling, G. P. Filipczyk, S. Petoud, Proc. SPIE. 2006, 6370, 6300Y-016300Y-11.

[23] J. P. Cross, M. Lauz, P. D. Badger, S. Petoud, J. Am. Chem. Soc. 2004, 126, 16278.

[24] V. Wintgens, P. Valat, J. Kossanyi, L. Biczok, A. Demeter, T. Berces, J. Chem. Soc., Faraday Trans. 1994, 90, 411.

[25] D. A. Chengelis, A. M. Yingling, P. D. Badger, C. M. Shade, S. Petoud, J. Am. Chem. Soc. 2005, 127, 16752.

[26] J. Zhang, C. M. Shade, D. A. Chengelis, S. Petoud, J. Am. Chem. Soc. 2007, 129, 14834.

[27] K. A. White, D. A. Chengelis-Czegan, M. Zeller, S. J. Geib, J. Szakos, S. Petoud, N. L. Rosi, Chem. Commun. 2009, 4506. 